\title{
Adapted NRC Based Sentiment Analysis in Event Distraction
}

\author{
Zeling WANG ${ }^{1}$, Bing WEI and Yibing LI \\ School of Computer Science and Technology, Hefei Normal University, Hefei, \\ 230601, China
}

\begin{abstract}
The paper was trying to extract entities from related tweets collected from twitter. This project first collected real-time tweets from twitter searching API with related topic-based hashtags during the death of American black man George Floyd. We then used two approaches to identify the polarities or emotions of each tweets and generated over-time sentiment flow chart in detecting entities. We found that some extreme sentiment score was correlated with some key entities over time. And our adapted NRC-lexicon based approach obtained better results. This paper revealed that public's sentiment displayed on tweets was generally consistent with the correlated events previously. It might help researchers in predicting or preventing public events in the future.
\end{abstract}

Keywords. Sentiment analysis, event distraction, social networking mining, NLP

\section{Introduction}

With the rapid development of online social networking, the analyzing of online social networking seems more popular for these decades. Many social networking users prefer to share their emotions such as joy, happiness, sadness, or sorrow on various social networking sites.

With more sharing information and opinions from users as well as the easy access API from various social networks, researchers nowadays can easily gather information to do some enterprise development, public sentiment surveys and customer satisfaction surveys. Sentiment analysis as a method using in social network analyzing, can be applied in different situations. For example, customers can judge a product before purchasing by the sentiment analysis of existing comments. Also, companies could use this sentiment research to find and analyze their brand influence among all their customers. In addition, the government or organizations can utilize this information to detect or prevent illegal issues before happening [1].

The experiment in this article is mainly done with Twitter. Users on twitter can create a short message called tweets, which can always represent different opinions or attitudes towards to various topics.

These years, sentiment analysis is widely applied in many areas in order to promote business sales or as a tool to collect customer's attitudes toward for later improvements. We are trying to calculate and analyze the sentiment change towards to one topic over time, to find some events or issues happening.

1 Corresponding Author, Zeling Wang, School of Computer Science and Technology, Hefei Normal University, Hefei,230601, China; E-mail: zw6e12@soton.ac.uk. 


\section{Background}

\subsection{Online Social Networking and Analysis}

With the development of World Wide Web, the social networking seems to be the key part in current internet. However, it is not coming directly from the born of World Wide Web. In Web 2.0, the Internet users become more important in contribution of the Internet. Everybody can be the content generator online, which gives the users brilliant ways to communicate and interact with each other [2,3].

With the analyzing of social networking graph, researchers can both improve the online system right now and try to find the deeper meaning from the large scale of information provided by online users [4].

\subsubsection{Micro Blog and Twitter}

Microblogging is an online phenomenon which gains popularity in recent years. It is a short blog or text (usually less than 200 words) to represent the users' status or lives. The text can be messaged to or interacted with their friends via text message, mobile apps or computer browsers. This type of microblogging service is provided by many companies (e.g. Twitter, Facebook). The most popular microblogging site is Twitter, which has many active users and tweets interacted online everyday [5]. On twitter, users form relationship with following and followed. Following a user means subscribing all the tweets they post. [6].

\subsubsection{Sentiment Analysis}

Sentiment analysis is a textual information mining based on a given texts. From Liu's research, the textual information can be categorized into 2 main types: facts and opinions. Facts are always the objective representation from the text, such as the events, activities or entities. Opinions mean the subjective representation from people, such as their sentiment, their mood, even their thought about some topics or entities. From the popularity of search engine, a lot of work about textual information has been done, such as text classification, text mining and natural language processing. Less focus is on the opinion analyzing; however, opinion is one of the most important element when people are judging or deciding. It may not only be used by individuals, but also the government or organizations $[3,7]$.

Due to the huge changes resulted from internet, the way how people represent their opinion changes a lot. People can easily express their feelings about some topics or events on forums, blogs or social networking sites. Those large amounts of information provide users and researchers a great chance to do the sentiment mining and analyzing work. For instance, if one prefers to buy some products, they can easily check the review or comments from other customers, instead of finding and asking their friends who have bought it before. In additional, with the collection of a large amount of sentiment data, the analyzing work not only give users or researchers great feedback, but also help us to identify or locating specific events about a topic, even it has been proved to complete some prediction work $[8,9,10]$. 


\section{Related work}

With the popularity of micro blog, many different approaches have been applied on the analysis of sentiment mining. However, the sentiment analysis on twitter is different from the sentiment analysis based on a given text, due to the limited length of a given tweet (140 characters), the irregular words in tweets and the creation of internet slang. Then, the data collected should always be cleaned up or processed in advance in order to generate high-quality result [5].

The study conducted by Go et al [11] applying emotion such as ":)" for positive ":(" for negative to classify the data. They first pre-processing the data and then conducted with three standard classifiers: multinomial Naïve Bayes, Maximum Entropy and Support Vector Machine (SVM). The best result is coming from the Maximus Entropy with approximately $83 \%$ accuracy with unigram and bigram together.

The approach used above did not identify and manage the neutral sentiment. The experiment did by Pak and Paroubek [12] is trying to improve. They collected the neutral tweets data from different newspaper and magazines, then with the three class NB classifier (positive, negative and neutral), which will be able to detect the neutral words from tweets data. However, this did not achieve an expected result. The accuracy of this experiment only comes to $40 \%$, however, the consideration of neutral would be useful and helpful in future opinion minding work.

From the idea coming from Speriosu et al [13], the sentiment analysis on twitter can be improved with the help of graph theories. They proposed that the combined sentiment label from propagation work can achieve a better accuracy in sentiment analysis [11].

Another research using a two-phased approach has been done by Barbosa and Feng [14]. The two-phases are separated the analyzing process into two steps. First step is to classify the tweets into objective or subjective class. Instead of the n-grams feature, they decided to use two different features "Meta-features" and "Syntax features".

additionally, the study from Agarwal et al. [15] shows a comparison of two different models based on their features and kernel trees. From their conclusion, they explored 50 different types of features in the experiment and found that both of their two different models from feature based and kernel tree based are better than the unigram baseline. Also, Kouloumpis [16] has tried to use variety of features such as n-gram features, lexicon features, part-of-speech features and micro-blogging features based on different corpus. The result comes from this experiment demonstrate that the micro-blogging features are clearly useful in sentiment classification of online tweets. However, other features are still required research or experiment.

Araque et al. [17] demonstrated that deep learning approach could be involved into sentiment analysis with the classification of different tweets. Apart from those traditional methods, Xiong [18] revealed a multi-layers sentiment-enriched word embedding methods, which involved asymmetric neutral layers can be greatly efficient in learning process in sentiment analysis. Reis [19] presented a random forest-based approach with the ROC curve and F1 score in detecting fake news online.

\subsection{Methodology}

The aim of this paper is to analyze the sentiment changes of a specific topic with hashtag in a given period (two weeks after George's death). With the death of black people George Floyd, there is a huge burst of tweets generated on twitter. The progress is shown 
in Figure 1. We first tried to collect the related tweets from different hashtags such as Floyd, WalkWithUs, BlackLivesMatter etc. in a consecutive period. After Analyzing the tweets with our two lexicons (The AFINN-111 and adapted NRC), we then tried to locate the specific events or sentiment burst from the sentiment changes overtime. The dataset used in the experiment is collected through Twitter's API with specific hashtag related to the topic.

The first step is to obtain the authentication from twitter. From the API correlated application, we can achieve some parameters in order to acquire the permission to collect data. Before the data is then stored in our csv files, we set up the hash tag (eg. WalkWithUs) to search and mine the tweets we expected with the given token.[20]

Data pre-process is done with some cleaning work since there are many noises affecting our result. Data cleaning [21] involves the removement of unnecessary data, such as Html tags, emojis, numbers etc.

After the preparation of raw data, the next step is to analyze and model the data. We first used the polarity-based corpus [13] to identify the sentiment of each tweet overtime. Then generated the charts to show the sentiment flow overtime to identify the burst or events. In this paper, the sentiment extraction was done with two approaches before comparison. One is using AFINN, which is a tweets-based lexicon with sentiment score ranging from -5 to 5 . The other is adapted NRC-lexicon, which included 8 different emotional categories.

When the sentiment detection was done, the next step is to generate the sentiment flow over time in the consecutive time-slots. The generated plots of sentiment fluctuation will be generated for us to identify the events.

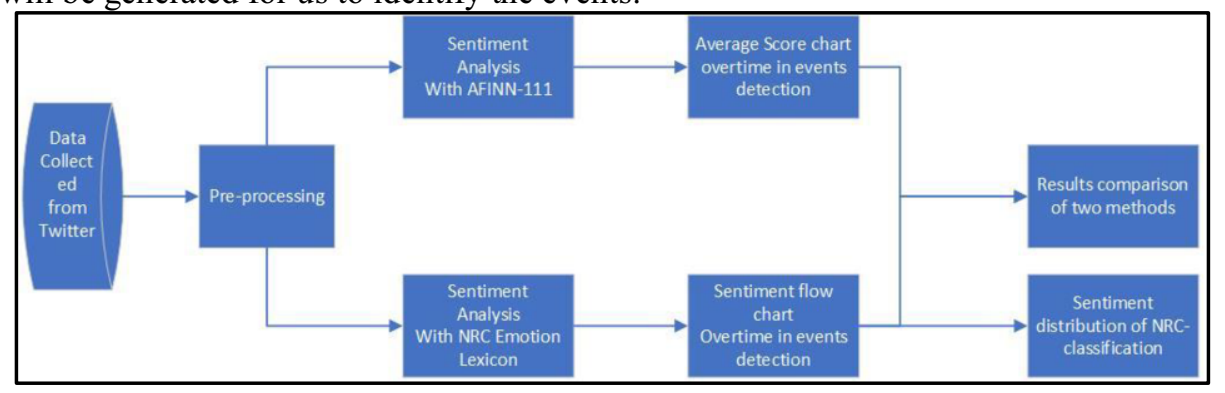

Figure 1. The Flow chart of the project.

\section{Implementation and Results}

The python was used to create the project. After first collecting data from the twitter API, we obtained the datasets for further sentiment analysis. We chose the Streaming API rather than the REST API, due to the REST one provides short period connection which is difficult to generate the continuous dataset. Then we used lexicon-based approach to analyze the sentiment for each time-slot (every minute). To fairly evaluate our approach, we also included the NRC dictionary to analyze and compare each result, the comparison would help us better insight the efficiency in sentiment output and event extraction [22]. 


\subsection{Lexicon Based Approach for Sentiment Classification}

The lexicon provides a series of labeled words or phrases for users to identify and classify the datasets. We conduct the sentiment analysis with the lexicon described above- the AFINN-111 [23]. Due to the flexibility and word-limit of online tweets, it is more suitable to choose a lexicon that constructed based on the tweets before. The AFINN111 is built by Nielsen with the tweets before, so that it is related to the language and structure of online tweets. AFINN-111 contains 2477 words with labels of sentiment strength from very negative $(-5)$ to very positive $(+5)$. In the experiment process, we apply the lexicon to analyze each tweet over time, and then we can generate the result with sentiment score of each tweet. This score intended to show the polarities of the tweets which can be utilized to monitor the sentiment change or flip over time [25]. The score is obtained from Eq. (1), in which the ps represents the positive score and pn means the number of the tweets, whereas the ns means negative score and nn stands for the number of related tweets.

$$
\text { Sentiment scores }=\frac{\sum p s * p n+n s * n n}{n}
$$

The key-word based sentiment classification approach is working in this way: in each tweet, the positive words and negative words found are count. Then by checking these keywords score from the lexicon, we can count the final score of each tweet. If the final score is larger than 0 , it means that the positive words are stronger than negative words in the tweet, so that the tweet is positive. By contrast, if the final score is less than 0 , which means that the negative words are stronger than the positive words in the tweet, the tweet will be classified as negative. However, if the final score equals 0 , it means that this is a neutral tweet or a tie.

\section{NRC Word-Emotion Association Lexicon}

The NRC Emotion Lexicon is a list of English words classified into 8 different emotions with simply 2 polarized sentiment positive and negative. The positive sentiment contains emotions of anticipation, trust, surprise and joy however the negative sentiment includes anger, fear, sadness and disgust.

The previous lexicon would count the words in the range of different sentiment scores, but due to the limitation of the size, the sentiment extraction would be restrained. However, the NRC Emotion Lexicon [24] is much more widely classified. With 8 different emotions, words and phrases can be better categorize. But it might receive better efficiency by add the factors in determine the polarity of each emotion.

From the related work done by Bandhakavi and Wiratunga [25], we realized that each emotion appeared in varied rates but represent different intensity. We then try to put coefficient for different emotion to receive better processing. We treat anger and disgust as extreme negative emotion by timing the count with 1.2. but sadness and fear as less-negative emotion, then times 0.8 . For the positive sentiments, we count joy and anticipation as extreme positive with timing 1.2, but surprise and joy as less-positive with the coefficient 0.8 . 
Sentiment score $=\frac{\sum_{n=1}^{8} \text { coefficient } * \text { count } * \text { score }}{\# \text { tweets number }}$

\section{Sentiment Analysis based on the events related to the death of George Floyd}

Tweets can always provide sentiment information while in the period or around a specific event. In this paper, we applied our discussed sentiment approaches to analyze the collected dataset and then tried to identify what these polarity information means and what they are associated with different entities [26]. On May 25th 2020, a 46-year-old black man, George Floyd died after officer kneels on his neck in custody. This event then trigger huge discussion on Twitter. We began to collect the tweets associated with this event during the next 2 weeks, and successfully identified the association between the sentiment changes correlated with the follow up events. The sentiment classification in 2 methods are showed in the figure 2. From the NRC-lexicon based approach [24], we identified 3 abnormal peaks in May 29th, June 1st and June 4th respectively. At the first week after George's death, the general emotional polarity of public is negative, but after June 1 st, especially the polarity began to flip to positive on 2nd June mainly because people began to protest peacefully in downtown Houston and government began to take the responsibilities of some issues. The 3 peak polarities are corelated with the events followed:

- May 29th: Officers arrested, charged in Floyd's death

- June 1st: Family autopsy revealed Floyd was asphyxiated by sustained pressure

- June 4th: Minneapolis memorial service for George Floyd honored his life, called Americans to action

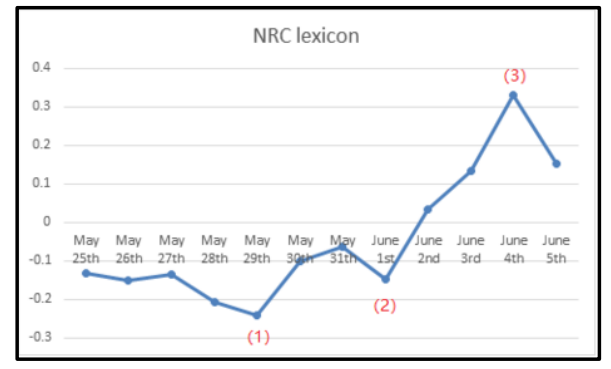

Figure 2. Sentiment score distribution from NRC approach.

Also, with the specific classification of 8 different emotions (Figure 3), we can then easily identify the tweets score of different categories, and then help us to understand the general public emotions towards the event.

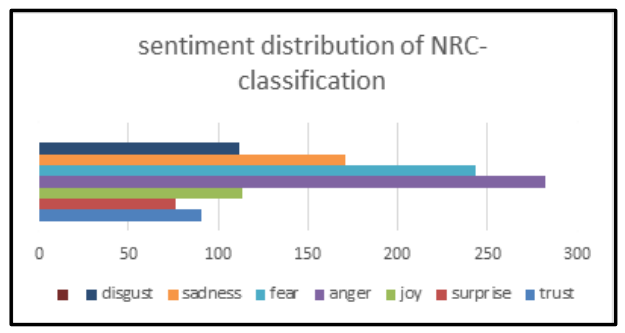

Figure 3. Categorized tweets in NRC approach. 
However, for the traditional lexicon AFINN-111(Figure 4), we still can identify the general trend that from negative gradually flip to positive. But the peak sentiment polarities are not as obvious as the NRC approach. It might due to the limitation amount of sentimental words included or because the words are only classified into 3 categories compared with 8 categories in NRC approach.

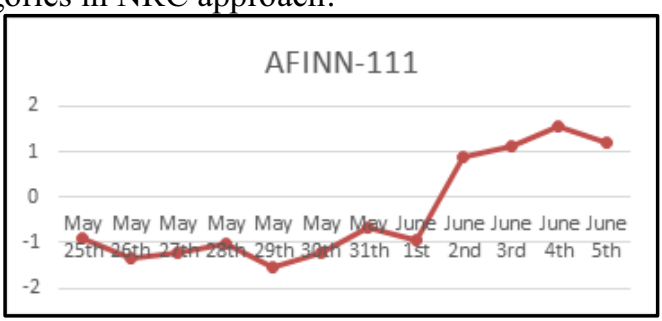

Figure 4. Sentiment score distribution from AFINN-111 approach.

\section{Future Work}

Future work will mainly try to deal with scalable amount of data for a specific event instead of the hashtag key words in twitter. While the dimension of data hugely increased to an event, the traditional lexicon-based analysis may be possible to combine with neural network. Apart from that, the coefficient we applied to modify the strength of positive and negative can be adjusted due to the general trends towards the event and it is important to find out the reliable coefficient in handling different issues.

\section{Conclusion}

We try to use the sentiment polarities in measuring the events happening from online social networking. From the approaches people talk about the events either positively or negatively, we figure out some correlations between the events happened at the same time. Analyzing tweets allowed us to extract related events and sentiment trends around the hot issues such as the public events followed with the death of George Floyd. In this paper, 2 lexicon-based approaches are used to classify the sentiment of our corpus. Moreover, we try to associate the polarities of tweets variation over time with the specific events happened incidentally. This approach of sentiment analysis helps us to extract events based on polarized-opinioned tweets. This may help the government or public organization in preventing the terrorism or avoiding the illegal events or protests in advance.

\section{Acknowledgement}

This research work is supported and funded by The Youth projects of Natural Science Foundation in Anhui Province(1908085QF287), and is also supported and funded by the Key projects of the National Natural Science Foundation of universities in Anhui Province (NO. KJ2020A0112, NO. KJ2018A0499) 


\section{References}

[1] Salloum SA, Al-Emran M, Monem AA, Shaalan K. A survey of text mining in social media: facebook and twitter perspectives. Adv. Sci. Technol. Eng. Syst. J. 2017 Jan; 2(1):127-33.

[2] O'reilly T. What is Web 2.0: Design patterns and business models for the next generation of software. Communications \& strategies. 2007 Mar 1(1):17.

[3] Mislove A, Marcon M, Gummadi KP, Druschel P, Bhattacharjee B. Measurement and analysis of online social networks. In Proceedings of the 7th ACM SIGCOMM conference on Internet measurement 2007 Oct 24 (pp. 29-42).

[4] Peng S, Wang G, Xie D. Social influence analysis in social networking big data: Opportunities and challenges. IEEE network. 2016 Nov 3; 31(1):11-7.

[5] Java A, Song X, Finin T, Tseng B. Why we twitter: understanding microblogging. In Proceedings of the 9th WebKDD and 1st SNA-KDD 2007 Workshop on Web Mining and Social Network Analysis 2018 (pp. 56-65).

[6] Kwak H, Lee C, Park H, Moon S. What is Twitter, a social network or a news media? In Proceedings of the 19th international conference on World wide web 2010 Apr 26 (pp. 591-600).

[7] Liu B. Sentiment analysis and subjectivity. Handbook of natural language processing. 2010 Feb; 2(2010): 627-66.

[8] Bakshi RK, Kaur N, Kaur R, Kaur G. Opinion mining and sentiment analysis. In2016 3rd International Conference on Computing for Sustainable Global Development (INDIACom) 2016 Mar 16 (pp. 452455). IEEE.

[9] Li N, Wu DD. Using text mining and sentiment analysis for online forums hotspot detection and forecast. Decision support systems. 2010 Jan 1;48(2):354-68.

[10] Lee D, Jeong OR, Lee SG. Opinion mining of customer feedback data on the web. In Proceedings of the 2nd international conference on Ubiquitous information management and communication 2008 Jan 31 (pp. 230-235).

[11] Go A, Bhayani R, Huang L. Twitter sentiment classification using distant supervision. CS224N project report, Stanford. 2009 Dec;1(12):2009.

[12] Pak A, Paroubek P. Twitter as a corpus for sentiment analysis and opinion mining. InLREc 2010 May 17 10(2010): 1320-1326.

[13] Speriosu M, Sudan N, Upadhyay S, Baldridge J. Twitter polarity classification with label propagation over lexical links and the follower graph. In Proceedings of the First workshop on Unsupervised Learning in NLP 2011 Jul (pp. 53-63).

[14] Barbosa L, Feng J. Robust sentiment detection on twitter from biased and noisy data. In Coling 2010: Posters 2010 Aug (pp. 36-44).

[15] Agarwal A, Xie B, Vovsha I, Rambow O, Passonneau RJ. Sentiment analysis of twitter data. In Proceedings of the workshop on language in social media (LSM 2011) 2011 Jun (pp. 30-38).

[16] Kouloumpis E, Wilson T, Moore J. Twitter sentiment analysis: The good the bad and the omg! InFifth International AAAI conference on weblogs and social media $2011 \mathrm{Jul} 5$.

[17] Symeonidis S, Effrosynidis D, Arampatzis A. A comparative evaluation of pre-processing techniques and their interactions for twitter sentiment analysis. Expert Systems with Applications. 2018 Nov 15; 110:298-310.

[18] Xiong S, Lv H, Zhao W, Ji D. Towards Twitter sentiment classification by multi-level sentiment-enriched word embeddings. Neurocomputing. 2018 Jan 31; 275: 2459-66.

[19] Reis JC, Correia A, Murai F, Veloso A, Benevenuto F. Supervised learning for fake news detection. IEEE Intelligent Systems. 2019 May 8;34(2):76-81.

[20] Trupthi M, Pabboju S, Narasimha G. Sentiment analysis on twitter using streaming API. In2017 IEEE 7th International Advance Computing Conference (IACC) 2017 Jan 5 (pp. 915-919). IEEE.

[21] Saif H, Fernández M, He Y, Alani H. On stopwords, filtering and data sparsity for sentiment analysis of twitter.

[22] Nausheen F, Begum SH. Sentiment analysis to predict election results using Python. In2018 2nd international conference on inventive systems and control (ICISC) 2018 Jan 19 (pp. 1259-1262). IEEE.

[23] Miura Y, Sakaki S, Hattori K, Ohkuma T. Team X: A sentiment analyzer with enhanced lexicon mapping and weighting scheme for unbalanced data. In Proceedings of the 8th International Workshop on Semantic Evaluation (SemEval 2014) 2014 Aug (pp. 628-632).

[24] Mohammad SM, Turney PD. NRC emotion lexicon. National Research Council, Canada. 2013 Nov 15;2.

[25] Bandhakavi A, Wiratunga N, Massie S, Padmanabhan D. Lexicon generation for emotion detection from text. IEEE intelligent systems. 2017 Feb 13;32(1):102-8.

[26] Yu Y, Wang X. World Cup 2014 in the Twitter World: A big data analysis of sentiments in US sports fans' tweets. Computers in Human Behavior. 2015 Jul 1; 48:392-400. 